\title{
Emerging Role of Safinamide in Parkinson's Disease Therapy
}

\author{
Jaime Kulisevsky $1,2,3$
}

1. Movement Disorders Unit, Neurology Department, Sant Pau Hospital, Autonomous University of Barcelona, Barcelona, Spain; 2. Estudis de Ciències de la Salut, Universitat Oberta de Catalunya (UOC), Barcelona, Spain; 3. CIBERNED, Instituto de Salud Carlos III, Madrid, Spain

\begin{abstract}
Parkinson's disease is associated with motor complications, especially dyskinesias, which limit dopaminergic replacement therapy. Safinamide is a water-soluble, orally active $\alpha$-aminoamide derivative that modulates dopaminergic and glutamatergic neurotransmission with a unique dual mechanism of action. It improves motor symptoms, motor complications, quality of life and 'on' and 'off' time even in combination with other Parkinson's disease (PD) medications, such as dopamine agonists and levodopa (LD). Safinamide reduces 'off' time and extends 'on' time without troublesome dyskinesia. Furthermore, safinamide maintains its effects after long-term treatment and has a favourable pharmacokinetic and side-effect profile. It therefore has the potential to become an important drug in PD management. This review will summarise data from animals, healthy human subjects and patients with PD on the long-term efficacy and safety of safinamide.
\end{abstract}

\section{Keywords}

Add-on therapy, dyskinesia, dopaminergic, monoamine oxidase b, non-dopaminergic, Parkinson's disease, safinamide

Disclosure: Jaime Kulisevsky has received honoraria for lecturing or participating in advisory boards of Abbvie, Lundbeck, Teva, UCB and Zambon, and research support from Instituto de Salud Carlos III, Spain.

Acknowledgements: Editorial assistance was provided by Catherine Amey at Touch Medical Media, London.

Received: 1 August 2014 Accepted: 22 August 2014 Citation: European Neurological Review, 2014;9(2):108-112 DOl:10.17925/ENR.2014.09.02.108

Correspondence: Jaime Kulisevsky, Sant Pau Hospital, Sant Antoni M Claret 167, 08025 Barcelona, Spain. E: jkulisevsky@santpau.cat

Support: The publication of this article was supported by Zambon SpA. The views and opinions expressed are those of the author and do not necessarily reflect those of Zambon SpA.

Parkinson's disease (PD) is a neurodegenerative disorder characterised by the loss of dopaminergic neurons in the substantia nigra pars compacta - non-dopaminergic neurotransmission is also involved. In the basal ganglia circuitry there are many non-dopaminergic neurotransmitters and neuromodulators involved in the control of motor symptoms and implicated in the development of motor complications following long-term levodopa (LD) therapy. ${ }^{1-4}$ Indeed, PD is no longer seen purely as a disease of the dopaminergic system. ${ }^{5}$ In particular, overactive glutamate transmission plays a role in the progression of $\mathrm{PD} .6-8$ Targeting non-dopaminergic systems is thus a complementary approach to improve and control such motor complications, removing the need for further increases in LD, which may otherwise worsen motor fluctuations. ${ }^{4}$

Safinamide (see Figure 1) is an oral, once-a-day adjunctive therapy developed for any stage of PD. Safinamide is a unique molecule with novel mechanisms of action (dopaminergic and non-dopaminergic) that include monoamine oxidase-B (MAOB) inhibition, sodium channel blockade and calcium channel modulation, thus inhibiting the excessive glutamate release. The sodium channel inhibition is concentration- and state-dependent, and does not influence physiological activity, avoiding depressant effects on the central nervous system (CNS). Safinamide does not affect L-type calcium channels (no effects in blood pressure and heart rate). ${ }^{9-12}$

Safinamide is more selective for MAO-B versus MAO-A than selegiline and rasagiline: 1,000-fold in humans, compared with 127-fold for selegiline and 103-fold for rasagiline. ${ }^{10,13-15}$ This higher selectivity is related to the absence of diet restrictions pertaining to the clinical use of the drug. Moreover, the MAO-B inhibition is totally reversible, allowing better clinical manageability and limiting possible drug-drug interactions. 10,14,15 $^{2}$

In animal models, safinamide has been shown to reduce LD-induced dyskinesias. ${ }^{10,16}$ It may also have neuroprotective effects. ${ }^{8}$ Safinamide inhibits $\alpha-1$ receptors in the endoplasmic reticulum. ${ }^{17}$ These receptors are believed to be multifunctional regulatory proteins with a role in CNS development, plasticity and neurodegeneration. Safinamide has been shown to completely prevent forebrain dopamine depletion and neuronal cell death in the gerbil substantia nigra when administered prior to 1-methyl-4-phenyl-1,2,3,6-tetrahydropyridine. ${ }^{18}$ In animal epilepsy models, safinamide was shown to counteract neuronal death that had been induced by excitotoxin. ${ }^{19}$ Possible mechanisms of action for safinamide's potential neuroprotective properties are MAO-B inhibition ${ }^{20}$ and reduction in glutamate release. ${ }^{10,11}$

The efficacy and safety of safinamide has been evaluated in clinical studies as add-on therapy to dopamine agonists (DAs) in early-stage $\mathrm{PD}^{21-23}$ and as add-on to LD (with possibly other anti-Parkinson's drugs) in mid- to late-stage PD. ${ }^{24-26}$

\section{Evidence from Healthy Volunteers}

Four clinical trials in male, healthy volunteers, which included a food interaction trial, covering doses from $25 \mu \mathrm{g} / \mathrm{kg}$ to $10 \mathrm{mg} / \mathrm{kg}$ were carried out to investigate the pharmacokinetics, pharmacodynamics and tolerability of safinamide, administered in single or repeated doses to steady state..$^{27}$ The trials showed that near complete inhibition of MAO-B is achieved with a safinamide dose of $0.3 \mathrm{mg} / \mathrm{kg}$ and that enteral absorption of the drug is linear and proportional to the doses 
administered. The drug is cleared from the body with a half-life $\left(t_{1 / 2}\right)$ approximately equal to $22 \mathrm{~h}$, without producing any clinically relevant accumulation at steady state. No evident MAO-A inhibition was observed at doses up to $10 \mathrm{mg} / \mathrm{kg}$.

In an investigation of the absorption, disposition, elimination and metabolic pathways, a single oral dose of $400 \mathrm{mg}{ }^{14} \mathrm{C}$-safinamide (as methanesulphonate salt), uniformly labelled in metabolically stable positions, was administered to healthy volunteers. ${ }^{28}$ Six male, nonsmoking healthy male volunteers were included. Maximum concentration was achieved at $1 \mathrm{~h}$ (plasma, median $\mathrm{T}_{\max }$ ) for parent drug and at 7 and $1.5 \mathrm{~h}$ for plasma and whole blood ${ }^{14} \mathrm{C}$ radioactivity, respectively. Terminal $t_{1 / 2}$ was about $22 \mathrm{~h}$ for unchanged safinamide. By contrast, ${ }^{14} \mathrm{C}$ radioactivity decreased in a biphasic manner with a terminal $t_{1 / 2}$ of $80 \mathrm{~h}$. The differences in $\mathrm{t}_{1 / 2}$ observed between parent safinamide and ${ }^{14} \mathrm{C}$ radioactivity in plasma can be explained by the presence of a varying mixture of metabolites that are cleared from the body compartments at different rates. Safinamide deaminated acid and the $\mathrm{N}$-dealkylated acid were identified as major metabolites in urine and plasma. These results indicated that safinamide can be administered as a single daily dose.

Non-selective MAO inhibitors or MAO A inhibitors can lead to a lifethreatening hypertensive crisis through the potentiation of peripheral adrenergic stimulation caused by tyramine ingested with food such as seasoned cheese or chicken liver, i.e. the 'cheese effect'. ${ }^{29}$ To assess the risk of inducing this cheese effect, the effect of safinamide on the pressor response to tyramine was compared with placebo in healthy male volunteers. ${ }^{30}$ The amount of tyramine needed to reach a blood pressure increase was the same after safinamide $2 \mathrm{mg} / \mathrm{kg}$ oral load versus placebo, suggesting that dietary restrictions for food high in tyramine should not be necessary with safinamide treatment. Furthermore, in healthy volunteers safinamide $300 \mathrm{mg}$ administered o.d. under fasting conditions did not change the tyramine pressor response at steady state after 6-7 days of treatment. ${ }^{31}$

\section{Evidence Base from Clinical Trials of Early Parkinson's Disease}

A dose-finding phase II study was carried out in 172 patients with early $\mathrm{PD}$, of whom four were not treated: 56 were randomised to placebo; 56 to safinamide $0.5 \mathrm{mg} / \mathrm{kg}$; 56 to safinamide $1 \mathrm{mg} / \mathrm{kg}$ (Study 009). ${ }^{32}$ Patients who improved their motor scores, as measured by the Unified Parkinson's Disease Rating Scale [UPDRS] III, by $\geq 30 \%$ after 3 months compared with baseline were classified as responders. The median daily administered safinamide dose in the $0.5 \mathrm{mg} / \mathrm{kg}$ group was $40 \mathrm{mg}$ (range: $20-40 \mathrm{mg}$ ) and $70 \mathrm{mg}$ (range: $40-90 \mathrm{mg}$ ) in the $1.0 \mathrm{mg} / \mathrm{kg}$ group. A responder rate of $37.5 \%$ was reported for patients in the higher safinamide dose group compared with $21.4 \%$ for placebo $(p<0.05)$.

A sub-analysis was performed in DA-treated patients $(n=101)$. A responder rate of $47.1 \%$ was achieved in the $1.0 \mathrm{mg} / \mathrm{kg}$ safinamide dose group compared with a $20.6 \%$ responder rate with placebo. The $1.0 \mathrm{mg} / \mathrm{kg}$ dose induced an average UPDRS-III improvement of 4.7 points compared with 1.4 points for placebo $(p<0.05)$.

In patients with early-stage PD, the efficacy and safety of 50-100 mg/ day or 150-200 mg/day safinamide in comparison with placebo were evaluated as add-on therapy to a single stable dose of DA (Study 015; NCT00643045). The primary endpoint was the change in UPDRS part III motor scores relative to baseline. ${ }^{21}$ In total, 269 patients were enrolled. Mean improvements from baseline to week 24 in UPDRS III total scores
Figure 1: Chemical Structure of Safinamide<smiles>C[C@H](NCc1ccc(OCc2cccc(F)c2)cc1)C(N)=O</smiles>

\section{Figure 2: MOTION Study}

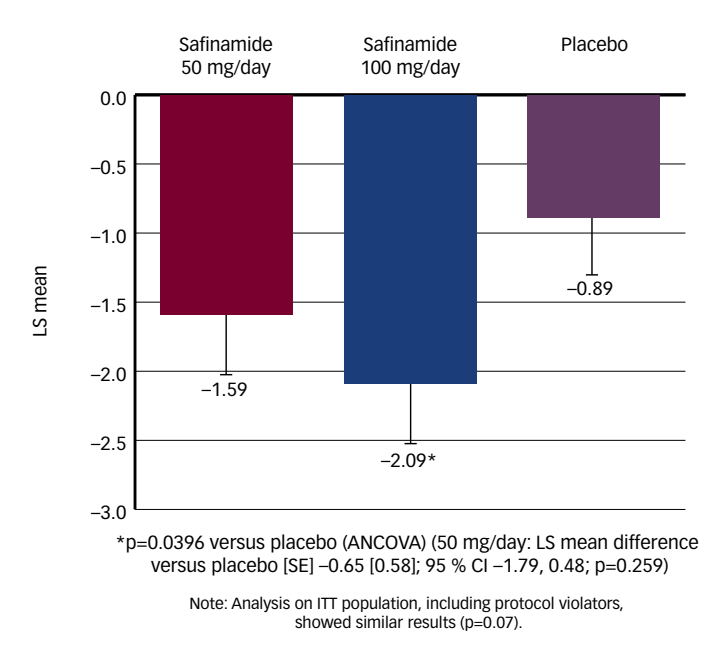

Change from baseline at week 24 in Unified Parkinson Disease Rating Scale (UPDRS) III total score in early PD with safinamide $50 \mathrm{mg} /$ day, safinamide $100 \mathrm{mg}$ /day and placebo in on-treatment, intent-to-treat (ITT) dopamine-agonist monotherapy population. ANCOVA = analysis of covariance; $\mathrm{Cl}=$ confidence interval; $L S=$ least squares; $S E=$ standard error.

were -3.90 for safinamide $200 \mathrm{mg},-6.0$ for safinamide $100 \mathrm{mg}$ and -3.60 for placebo. The difference between $100 \mathrm{mg} /$ day and placebo was significant (point estimate: $-1.9 ; 95 \%$ confidence interval [Cl] -3.7 to $-0.1 ; p=0.0419$ ). No clinically meaningful differences in comparison with placebo were observed for any safety variables.

The extension study to Study 015 (Study 017, NCT00642889) was a 52week, randomised, double-blind, placebo-controlled pre-planned study to investigate the long-term efficacy and safety of safinamide as addon therapy for early PD. The primary efficacy endpoint was the time from baseline (Study 015 randomisation) to 'intervention', defined as: increase in DA dose; addition of another DA, LD or other PD treatment; or discontinuation due to lack of efficacy.22 The pooled data from the safinamide groups did not show a statistically significant difference versus placebo for the primary endpoint of median time from baseline to intervention. This may have been due to a lack of response in patients receiving the higher dose of safinamide (150-200 mg/day). ${ }^{15} \mathrm{~A}$ post-hoc analysis revealed that patients who had received safinamide $100 \mathrm{mg} /$ day experienced a significantly lower rate of interventions compared with patients receiving DA monotherapy (25\% versus $51 \%$, respectively) and delay in median time to intervention of 9 days $(\mathrm{p}<0.05 ; 240-540-$ day analysis). No new safety concerns emerged with safinamide in this study.22

The MOTION study (NCT00605683) is a 24-week randomised placebocontrolled trial evaluating the safety and efficacy of oral $50 \mathrm{mg} /$ day and $100 \mathrm{mg} /$ day of safinamide as add-on to stable DA monotherapy and who were LD naive. ${ }^{23}$ The study randomised 679 patients with 


\section{Figure 3: Mean Change from Baseline in Total Daily on Time without Troublesome Dyskinesia in the SETTLE Study}

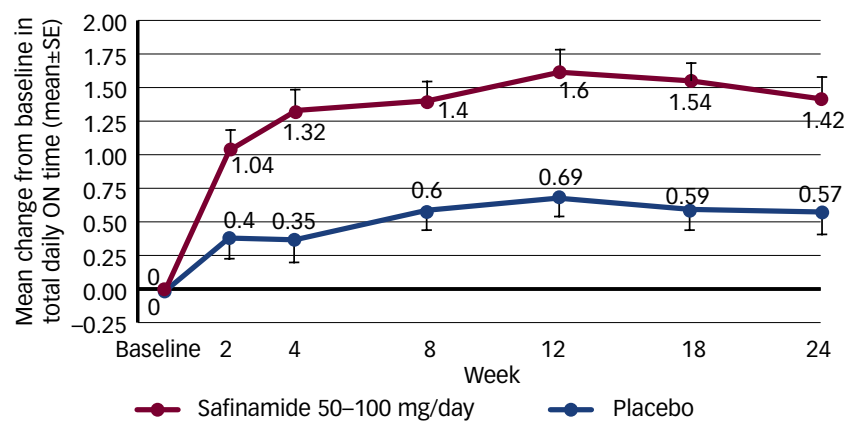

$S E=$ standard error

\section{Figure 4: Primary Endpoint from Study 016}

$\begin{array}{lcc} & \text { Safinamide } 50 \mathrm{mg} & \text { Safinamide } 100 \mathrm{mg} \\ \text { LS mean } & 1.23 & 1.28 \\ \text { LS difference versus placebo } & 0.51 & 0.55 \\ 95 \% \mathrm{Cl} \text { or LS difference } & (0.07,0.94) & (0.12,0.99) \\ \text { p value versus placebo } & 0.0233 & 0.0130\end{array}$

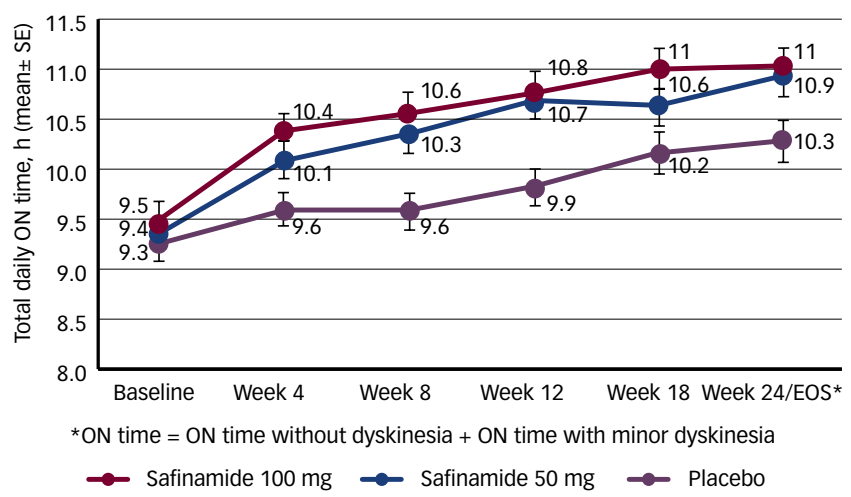

Mean change standard error (SE) in on time with no or minor dyskinesia (patient diary data). Using analysis of covariance (ANCOVA) analysis (mixed model repeated measures [MMRM]), all time points after baseline were statistically significant compared with placebo, with the exception of safinamide $50 \mathrm{mg}$ /day at week $18(p=0.0974)$. ${ }^{*} p<0.05$ versus placebo. $\mathrm{Cl}=$ confidence interval; $E O S=$ end of study; $L S=$ least squares.

early PD (i.e. diagnosed with PD within 5 years); 666 of whom were receiving dopamine-agonist monotherapy (safinamide $50 \mathrm{mg} / \mathrm{day}$, $\mathrm{n}=223$; safinamide $100 \mathrm{mg} /$ day, $\mathrm{n}=221$; placebo, $\mathrm{n}=222$ ). Safinamide $100 \mathrm{mg} /$ day significantly improved motor symptoms (as assessed using UPDRS III; $p=0.04$ ) (see Figure 2). Safinamide $100 \mathrm{mg} /$ day also significantly improved quality of life (as assessed using EuroQoL [EQ5D] and Parkinson's Disease Questionnaire [PDQ-39]) compared with placebo in patients taking a single DA $(n=666)$. These results further support safinamide, at a $100 \mathrm{mg} /$ day dose, as an efficacious add-on treatment in early-stage PD.

\section{Evidence Base from Clinical Trials of Mid- to Late-stage Parkinson's Disease}

The SETTLE trial (NCT00627640) was a 6-month (24-week), randomised, double-blind, placebo-controlled trial that evaluated the efficacy and safety of a dose range of safinamide (50-100 $\mathrm{mg}$ ) versus placebo as add-on therapy to a stable dose of LD and other anti-Parkinson drugs in patients with mid- to late-stage idiopathic PD (i.e. diagnosed with PD more than 5 years earlier). ${ }^{24}$ In the SETTLE trial, 549 patients with advanced idiopathic PD and motor fluctuations were randomised: 274 to safinamide; 275 to placebo.

The primary efficacy measure was the change from baseline to 24 weeks in daily 'on' time without troublesome dyskinesia as recorded by patients or caregivers in an 18-hour diary. Responders were taken to be patients with clinically relevant improvements from baseline in motor fluctuations ('on' and 'off' time, as determined from the patient diary) and in motor function (UPDRS III score) at 24 weeks. The analyses of clinical relevance were performed using cut-off of 30 and 60 minutes for motor fluctuations, $20 \%$ and $30 \%$ improvement in motor symptoms. In particular, clinically relevant improvements in motor fluctuations were defined by a change $\geq 30$ and $\geq 60$ minutes in 'on' time (increase) and 'off' time (decrease) at 24 weeks versus baseline. Clinically relevant improvements in motor function were defined by $a \geq 20 \%$ and $a \geq 30 \%$ decrease in the UPDRS III score at 24 weeks compared with baseline. At 24 weeks, the mean ( \pm Standard deviation [SD]) change in daily 'on' time from baseline for the safinamide group was $1.42( \pm 2.80)$ hours, whereas the placebo group had a mean $( \pm S D)$ change of $0.57( \pm 2.47)$ hours $(p<0.001)$ (see Figure 3$).^{24}$

Significantly more patients in the safinamide group (59/274, $21.5 \%)$ than in the placebo group $(37 / 275,13.5 \%)$ had an improvement $\geq 30$ minutes in both 'on' and 'off' time and a $\geq 20 \%$ improvement in the UPDRS III score, from baseline to 24 weeks (odds ratio 1.77, $95 \% \mathrm{Cl} 1.13-2.77 ; p=0.0134$ ). The difference between treatment groups in the rates of patients achieving clinically relevant results was also significant when defined by more stringent criteria: a total of 37/274 (13.5\%) patients treated with safinamide versus 20/275 (7.3\%) patients in the placebo group had their on and off time improved by $\geq 60$ minutes with an improvement in the UPDRS III score $\geq 30 \%$ (odds ratio $2.00,95 \% \mathrm{Cl} 1.13-3.55$; $\mathrm{p}=0.0180$ ). ${ }^{24}$ The significant benefit of safinamide over placebo in patients with advanced PD observed in the SETTLE study was therefore clinically relevant as it allowed more patients to achieve improvements of at least 1 hour in motor fluctuations and a percentage of improvement of $30 \%$ and more in motor symptoms.

Study 016 (NCT01187966) is a phase III, multicentre, double-blind, placebo-controlled, parallel group study that evaluated the efficacy and safety of safinamide as add-on to LD in the treatment of patients with PD and motor fluctuations. ${ }^{25}$ The primary endpoint was the change in daily 'on' time with no or non-troublesome dyskinesia, as recorded by patients' diaries. Secondary endpoints included 'off' time, UPDRS part III (motor score) and the Clinical Global Impression scale (CGI-Change).

In Study 016, 669 patients with idiopathic PD on a stable dose of LD therapy were randomised to receive $50 \mathrm{mg} /$ day safinamide $(n=223)$, $100 \mathrm{mg} /$ day safinamide $(n=224)$ or placebo $(n=222)$ for 6 months. The 6 months of treatment with safinamide ( 50 or $100 \mathrm{mg} /$ day) compared with placebo significantly improved motor function, increased the average duration of 'on' time with no dyskinesia or with minor dyskinesia and decreased the average duration of the 'off' time (see Figure 4). Patients considered 'on' time with no or non-troublesome dyskinesia as 'good' on time correlated with a good response to the treatment throughout the day. Moreover, both safinamide doses did not increase the troublesome dyskinesia despite the significant increase in 'on' time. ${ }^{25}$

Study 018 (NCT01286935) was an 18-month, placebo-controlled extension to Study 016 that aimed to assess the long-term safety and efficacy of safinamide as add-on to LD in patients with PD and motor fluctuations. ${ }^{26}$ In total, $81.3 \%$ (544 out of 669) patients initially randomised 
for study 016 entered this extension trial. The primary endpoint was the change from baseline in Dyskinesia Rating Scale (DRS) ${ }^{33}$ total score during 'on' time over 24 months. Other efficacy endpoints included the change in 'on' time without troublesome dyskinesia, changes in individual diary categories, depressive symptoms and quality of life measures. At month 24 , there was no significant difference in the primary endpoint between safinamide and placebo, despite observed mean DRS decreases of $31 \%$ and $27 \%$, respectively, for $50 \mathrm{mg} /$ day and $100 \mathrm{mg} /$ day; whereas the other efficacy measures improved. However, baseline DRS scores were Iow (the mean DRS score was lower than 4) and this may have accounted for this finding. In a post hoc analysis of the subgroup of patients with DRS $>4$ (a value considered clinically relevant), safinamide $100 \mathrm{mg} /$ day improved DRS scores versus placebo $(p=0.0317)$.

Improvements observed at week 24 of study 016 in motor function, quality of life, 'on' time with no or non-troublesome dyskinesia and 'off' time, were confirmed after 24 months. ${ }^{26}$ This can be considered as further evidence that safinamide improves motor fluctuations without worsening dyskinesia, maintaining its efficacy in the long term (see Figure 5). Both doses were well tolerated during 2 years of treatment, with a similar incidence of adverse events versus placebo.

The clinical studies in patients with mid- to late-stage PD ${ }^{24,26}$ thus support the use of safinamide $100 \mathrm{mg} /$ day as add-on treatment to LD and other anti-Parkinsonian drugs.

\section{Summary of Safety and Tolerability}

common side effects (reported in $\geq 5 \%$ of patients in any group) included worsening of Parkinson's disease, cataract, back pain, pyrexia and hypertension. ${ }^{22,25}$ No clinically significant differences between safinamide and placebo were observed for any safety variables in the clinical trials of safinamide. 12,22-26,32 In Study 015, the incidence of treatment-emergent adverse events (TEAES) was $<10 \%$ for each group and the number of patients with TEAEs rated as severe was $2.2 \%$ for the safinamide $100 \mathrm{mg}$ group, $10.1 \%$ for the safinamide $200 \mathrm{mg}$ group and $6.7 \%$ for placebo. ${ }^{21}$ Incidences of serious drug-related TEAS in Study 017 were $7.5 \%$ for the safinamide $100 \mathrm{mg}$ /day group, $1.4 \%$ for safinamide $200 \mathrm{mg} /$ day and $5.1 \%$ for placebo. ${ }^{22}$ The incidence of TEAEs, both newly emergent and re-emergent, in the combined 016 and 018 study population were likewise similar across groups. Number of patients experiencing any serious TEAEs in the placebo group were $16 \%, 16.9 \%$ in the safinamide $50 \mathrm{mg}$ /day group and $18.9 \%$ in the safinamide $100 \mathrm{mg} /$ day group. No dose response for safinamide on the frequency of AES has been reported although further study is still needed.

\section{Conclusions}

The cause of PD is multifactorial and the pathomechanism of the disease is not completely understood. The basal ganglia circuitry involves many non-dopaminergic neurotransmitters and neuromodulators in the control of motor symptoms and in the development of motor complications following long-term LD therapy. In particular, overactive glutamate transmission plays a role in the progression of PD. ${ }^{6-8}$ There is a need for new PD treatments combining dopaminergic and non-dopaminergic effects, able to prevent the onset of motor complications keeping unchanged the efficacy and with a simple, well-tolerated treatment regime.

Safinamide is a potent, highly selective, reversible MAO-B inhibitor with anti-glutamatergic effects. It blocks fast sodium currents and modulates $\mathrm{N}$-type calcium currents in a concentration- and state-dependent manner

\section{Figure 5: Principal Secondary Endpoint from Study 018}

\begin{tabular}{lcc}
\hline & Safinamide $50 \mathrm{mg}$ & Safinamide $100 \mathrm{mg}$ \\
LS mean $(\mathrm{h})$ & 1.01 & 1.18 \\
LS difference versus placebo $(\mathrm{h})$ & 0.67 & 0.83 \\
$95 \% \mathrm{Cl}$ or LS difference $(\mathrm{h})$ & $(0.23,1.11)$ & $(0.39,1.27)$ \\
p value versus placebo & 0.0031 & 0.0002
\end{tabular}

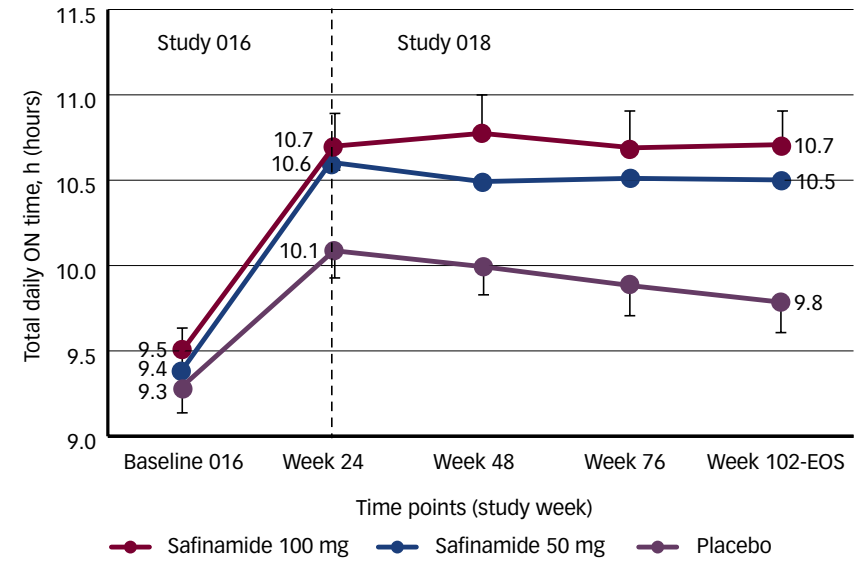

Mean change in 'ON' time without troublesome dyskinesia over 2 years (from baseline of Study 016 to end of Study 018 - patient diary data). $\mathrm{Cl}=$ confidence interval; EOS = end of study; $L S=$ least squares.

(different potency at different states of the channel), thus inhibiting the presynaptic release of excitotoxic amino acids like glutamate.

Safinamide does not affect L-type calcium channels (no effects in blood pressure and heart rate) and can be given without food restrictions related to tyramine. Moreover, safinamide has a dose-dependent, doseproportional and linear favourable pharmacokinetic profile.

Safinamide was investigated in phase III clinical trials as adjunct therapy to DAs for patients with early stage PD and to LD therapy for patients in the mid-to-late stages of PD. Safinamide, at a $100 \mathrm{mg} /$ day dose, improves fluctuations and controls motor symptoms and motor complications in the short term maintaining the benefits in the long term (up to 2 years). Results from long-term (24 months) double-blind controlled studies suggest that safinamide does not increase the risk of developing troublesome dyskinesia despite significantly increasing the daily 'on' time. Importantly, long-term results suggest that safinamide treatment improves dyskinesia in patients with moderate to severe dyskinesia. Safinamide reduces daily 'off' time and early morning akinesia, improving quality of life and functionality and significantly improves in the long-term motor functions as evaluated by UPDRS III score. It is well tolerated with a favourable side-effect profile and is easy to use: once-daily dose, no need of LD adjustment, no major drugdrug interactions, no diet restrictions due to its higher MAO-B/MAO-A selectivity (1,000-fold more selective in humans for MAO-B compared with 127 for selegiline and 103 for rasagiline).

Safinamide, unlike other drugs that can improve motor function and act on the dopaminergic pathway in PD, does not aggravate dyskinesias. This positive effect may be related to its dual mechanism acting on both the dopaminergic and the glutamatergic pathways. Contrary to selegiline and rasagiline, which show dyskinesia as a secondary effect in patients with advanced PD, safinamide has no methamphetamine metabolites (i.e. in contrast to selegiline) ${ }^{34}$ and acts on the glutamatergic pathway (i.e. in contrast to rasagiline). 
Safinamide is a unique compound exhibiting a combined nondopaminergic and dopaminergic mode of action, which modulates altered dopaminergic and glutamatergic neurotransmission in PD patients; therefore, it has the potential to become an important drug for PD management, in all stages of the illness. More data, in particular, from independent clinical trials, are needed to assess the compound in more detail. Overall, preclinical and clinical data with safinamide support the view that it may serve as a valuable choice for add-on therapy to DAs and LD in the early and the mid-to-late stage disease, respectively.
1. Graybiel AM, Neurotransmitters and neuromodulators in the basal ganglia, Trends Neurosci, 1990;13,244-54.

2. Albin RL, Young AB, Penney JB, The functional anatomy of disorders of the basal ganglia, Trends Neurosci, 1995; 18:63-4

3. Braak H, Del Tredici K, Rub U, et al., Staging of brain pathology related to sporadic Parkinson's disease, Neurobiol Aging, 2003;24:197-211.

4. Fox SH, Non-dopaminergic treatments for motor control in Parkinson's disease, Drugs, 2013;73:1405-15.

5. Huot P, Fox SH, Nondopaminergic treatments for Parkinson's disease, Neurodegener Dis Manag, 2011;1:491-512.

6. Blandini F, Porter RH, Greenamyre JT, Glutamate and Parkinson's disease, Mol Neurobiol, 1996;12:73-94. Parkinson's disease, Mol Neurobiol, 1996;12:73-94.
Chase TN, Bibbiani F, Oh JD, Striatal glutamatergic mechanisms and extrapyramidal movement disorders, mechanisms and extrapyramidar

8. Borgohain R, Kandadai RM, Safinamide: a novel antiParkinsonian drug with multiple actions, Neurodegen Dis Manage 2013:3:231-40

9. Pevarello P, Bonsignori A, Dostert P, et al., Synthesis and anticonvulsant activity of a new class of 2-((arylalkyl) amino alkanamide derivatives, I Med Chem, 1998;41:579-90.

10. Caccia C, Maj R, Calabresi M, et al., Safinamide: from molecular targets to a new anti-Parkinson drug, Neurology, 2006;67(7 Suppl. 2):S18-23.

11. Caccia C, Salvati P, Rossetti S, Anand R, Safinamide: Modulation of dopaminergic and glutamatergic system, Mov Disord, 2008;67:S22-3

12. Chazot PL, Safinamide for the treatment of Parkinson's disease, epilepsy and restless legs syndrome, Curr Opin disease, epilepsy and restless
Investig Drugs, 2007:8:570-9.

13. Binda $C$, Wang J, Pisani $L$, et al., Structures of human monoamine oxidase B complexes with selective noncovalent inhibitors: safinamide and coumarin analogs, J Med Chem, 2007;50:5848-52.
14. Onofrj $\mathrm{M}$, Bonanni L, Thomas $\mathrm{A}$, An expert opinion on safinamide in Parkinson's disease, Expert Opin Investig Drugs, 2008;17:1115-25

15. Müller T, Current status of safinamide for the drug portfolio of Parkinson's disease therapy, Expert Rev Neurother 2013;13:969-77.

16. Grégoire $L$, Jourdain VA, Townsend $M$, et al., Safinamide reduces dyskinesias and prolongs I-DOPA antiparkinsonian effect in parkinsonian monkeys, Parkinsonism Relat Disord, 2013;19:508-14.

17. Fariello RG, MCArthur RA, Bonsignori A, et al., Preclinical evaluation of PNU-151774E as a novel anticonvulsant, J Pharmacol Exp Ther, 1998;285:397-403.

18. Vaghi F, Maj R, Rosa B, et al., Neuroprotective effect of PNU151774E, a new anticonvulsant compound, in the model of global ischaemia in gerbils, Soc Neurosci Abstr, 1997:23:545.

19. Maj R, Fariello RG, Ukmar G, et al., PNU-151774E protects against kainate-induced status epilepticus and hippocampal lesions in the rat, Eur J Pharmacol, 1998;359:27-32.

20. Kupsch A, Sautter J, Götz ME, et al., Monoamine oxidaseinhibition and MPTP-induced neurotoxicity in the non-human primate: comparison of rasagiline (TVP 1012) with selegiline, J Neural Transm, 2001; 108:985-1009.

21. Stocchi F, Borgohain R, Onofrj M, et al., A randomized, double-blind, placebo-controlled trial of safinamide as add-on therapy in early Parkinson's disease patients, Mov Disord 2012;27:106-12.

22. Schapira AH, Stocchi F, Borgohain R, et al., Long-term efficacy and safety of safinamide as add-on therapy in early Parkinson's disease, Eur / Neurol, 2013:20:271-80.

23. Anand $R$, Barone $P$, Fox $S$, et al., Safinamide is effective as add-on treatment in both early and advanced PD. Presented at the XXI World Congress of Neurology, Wien, Austria, 21-26 September 2013.

24. Schapira AH, FOX S, Hauser, et al., Safinamide significantly improves responder rates in fluctuating PD patients as add-on to levodopa (SETTLE). Presented at the 17th International Congress of Parkinson's Disease and Movement Disorders, Sydney, Australia, 16-20 June 2013

25. Borgohain R, Szasz J, Stanzione P, et al., Randomized trial of safinamide add-on to levodopa in Parkinson's disease with motor fluctuations, Mov Disord, 2014;29:229-37.

26. Borgohain R, Szasz J, Stanzione P, et al., Two-year, randomized, controlled study of safinamide as add-on to levodopa in mid to late PD, Mov Disord, 2014;29:1273-80.

27. Marzo A, Dal Bo L, Monti NC, et al., Pharmacokinetics and pharmacodynamics of safinamide, a neuroprotectant with antiparkinsonian and anticonvulsant activity, Pharmacol Res, 2004;50:77-85.

28. Leuratti C, Sardina M, Ventura P, et al., Disposition and metabolism of safinamide, a novel drug for Parkinson's disease, in healthy male volunteers, Pharmacology, 2013:92:207-16.

29. Walker SE, Shulman KI, Tailor SA, Gardner D, Tyramine content of previously restricted foods in monoamine oxidase inhibitor diets, I Clin Psychopharmacol, 1996:16:383-8.

30. Cattaneo C, Caccia C, Marzo A, et al., Pressor response to intravenous tyramine in healthy subjects after safinamide, a novel neuroprotectant with selective, reversible monoamin oxidase B inhibition, Clin Neuropharmacol, 2003;26:213-7.

31. Di Stefano AF, Rusca A, Pressor response to oral tyramine during co-administration with safinamide in healthy volunteers, Naunyn Schmiedebergs Arch Pharmacol, 2011;384:505-15

32. Stocchi F, Arnold G, Onofri M, et al., Improvement of motor function in early Parkinson disease by safinamide, Neurology, 2004;63:746-8.

33. Goetz CG, Stebbins GT, Shale HM, et al., Utility of an objective dyskinesia rating scale for Parkinson's disease: inter- and intrarater reliability assessment, Mov Disord, 1994:9:390-4.

34. Kalász H, Magyar K, Szke É, et al., Metabolism of selegiline [(-)-deprenyl)], Curr Med Chem, 2014;21(13):1522-30. 